\title{
Theory of the thermal expansion of Si and diamond
}

\author{
C. H. Xu, C. Z. Wang, C. T. Chan, and K. M. Ho \\ Ames Laboratory-U.S. Department of Energy, \\ and Department of Physics and Microelectronics Research Center, Iowa State University, Ames, Iowa 50011
}

(Received 24 September 1990)

\begin{abstract}
Phonon-dispersion curves, lattice mode Grüneisen parameters, and the coefficients of thermal expansion are calculated for $\mathrm{Si}$ and $\mathrm{C}$ within a tight-binding model. The results are in good agreement with experimental data. The origin of the negative thermal expansion in $\mathrm{Si}$ is examined, and we find that the different thermal-expansion behaviors between $\mathrm{Si}$ and $\mathrm{C}$ can be explained by the different relative strengths of bond-bending and bond-stretching forces.
\end{abstract}

Thermal expansion in semiconductors is a topic of some technological as well as scientific interest since it plays an important role in determining the residual stresses in solid-state devices. While most materials expand upon heating, many tetrahedral semiconductors (e.g., $\mathrm{Si}, \mathrm{Ge}$, and $\mathrm{GaAs}$ ) exhibit negative thermal expansion at low temperatures. ${ }^{1-4}$ This problem has received a fair amount of theoretical attention, mostly empirical studies relying heavily upon experimental input. ${ }^{5}$ An exception is the recent work of Biernacki and Scheffler, ${ }^{6}$ who calculated the negative thermal expansion of $\mathrm{Si}$ with a simple model based on two force constants extracted from local-density-functional calculations. It is generally believed that the unusual negative thermal-expansion behavior can be attributed to the negative Grüneisen parameters (i.e., phonon frequency increases as crystal volume increases) of the transverse acoustic (TA) phonons near the Brillouin-zone boundary, which have also been reproduced by first-principles theories. ${ }^{7,8}$ In this paper, we choose $\mathrm{Si}$ and $\mathrm{C}$ as prototypical tetrahedral semiconductor systems and demonstrate that a simple tightbinding model ${ }^{9}$ can describe the thermal-expansion behavior accurately in these systems without fitting to experimental information. The origin of the negative thermal-expansion behavior is explained with a simple physical model. The difference in thermal-expansion behavior between $\mathrm{Si}$ and $\mathrm{C}$ arises from the difference in the relative strengths of the central forces and angular forces in the crystal in agreement with ideas in previous investigations. $^{10}$

The free energy of a crystal can be expressed in the quasiharmonic approximation ${ }^{11}$ as

$$
\begin{aligned}
F(V, T)= & E(V)+\frac{1}{2} \sum_{i} h v_{i}(V) \\
& +k_{B} T \sum_{i} \ln \left\{1-\exp \left[-h v_{i}(V) / k_{B} T\right]\right\},
\end{aligned}
$$

where $E(V)$ is the energy of the static lattice with volume $V$, and $v_{i}(V)$ are the normal-mode frequencies of smallamplitude oscillations for the crystal at volume $V$.

To obtain the phonon frequencies $v_{i}$ at a given crystal volume, we employ a simple model in which the total energy of the crystal $E(V)$ is expressed as a sum of two terms: $E_{\mathrm{bs}}$, the electronic band-structure energy, is the sum of single-electron energies over all occupied electronic states, and $U=\frac{1}{2} \sum_{i, j} \phi\left(r_{i j}\right)$ is a sum of nearestneighbor pair interactions. The band structure is evaluated using an empirical tight-binding model following the previous work of Chadi and co-workers. ${ }^{12,13}$ The nearest-neighbor $s p^{3}$ parameters are determined by fitting the electronic bands of $\mathrm{Si}$ and $\mathrm{C}$ in diamond structures: $\varepsilon_{s}=-5.25 \mathrm{eV}, \quad \varepsilon_{p}=1.20 \mathrm{eV}, \quad V_{s s \sigma}=-1.938 \mathrm{eV}$, $V_{s p \sigma}=1.745 \mathrm{eV}, V_{p p \sigma}=3.05 \mathrm{eV}$, and $V_{p p \pi}=-1.075 \mathrm{eV}$ for $\mathrm{Si}$; and $\varepsilon_{s}=-2.99 \mathrm{eV}, \varepsilon_{p}=3.71 \mathrm{eV}, V_{s s \sigma}=-5.55 \mathrm{eV}$, $V_{s p \sigma}=5.91 \mathrm{eV}, V_{p p \sigma}=7.78 \mathrm{eV}$, and $V_{p p \pi}=-2.50 \mathrm{eV}$ for C. These parameters are scaled with interatomic separation $r$ as $r^{-2}$. Unlike the Chadi model, ${ }^{13}$ the short-ranged pair potential $\phi(r)$ in our calculation is determined ${ }^{9}$ by subtracting the $E_{\mathrm{bs}}$ contribution from first-principles local-density-functional results for the volume-dependent total energy of the crystal in the ideal diamond structure $^{8,14}$ Thus the parameters of our model can be extracted entirely from first-principles calculations without fitting to any experimental data.

The force-constant matrix is obtained by taking the second derivatives of the total energy with respect to the atomic displacements and, using second-order perturbation theory, can be expressed as

$$
\begin{aligned}
& K_{i \alpha, j \beta}= \frac{\partial^{2} U}{\partial x_{i \alpha} \partial x_{j \beta}} \\
&+2 \sum_{n \mathbf{k}, n^{\prime} \mathbf{k}^{\prime}} \frac{f_{n \mathbf{k}}-f_{n^{\prime} \mathbf{k}^{\prime}}}{\varepsilon_{n \mathbf{k}}-\varepsilon_{n^{\prime} \mathbf{k}^{\prime}}}\left\langle n \mathbf{k}\left|\frac{\partial^{2} H}{\partial x_{i \alpha} \partial x_{j \beta}}\right| n \mathbf{k}\right\rangle \\
& \\
& \times\left\langle n^{\prime} \mathbf{k}^{\prime}\left|\frac{\partial H}{\partial x_{i \alpha}}\right| n^{\prime} \mathbf{k}^{\prime}\right\rangle \\
&\left.\frac{\partial \mathbf{k}}{\partial \beta}\right\rangle,
\end{aligned}
$$

where $H$ is the tight-binding Hamiltonian, $|n \mathbf{k}\rangle$ are electronic states, and $f_{n \mathbf{k}}$ are Fermi occupation factors. The first term in Eq. (2) is the contribution from the shortranged pair potential. The second and the third terms represent the contributions from the electronic band structure.

Using the above force-constant matrices, we can evaluate the dynamical matrix ${ }^{15}$ for any wave vector $q$. We 
obtained the phonon frequencies on a grid of 240 wave vectors in the irreducible part of the Brillouin zone. The calculated dispersion curves for $\mathrm{Si}$ and $\mathrm{C}$ are compared with experimental data in Fig. 1. Good agreements are achieved for the acoustic phonon modes. Our model tends to overestimate the optical-mode frequencies by $\sim 10 \%$. Using the calculated phonon frequencies, $F(V, T)$ is evaluated for a set of volumes and temperatures. The equilibrium volumes at various temperatures are determined by minimizing $F(V, T)$ as a function of $V$. The results for the linear expansion coefficients of $\mathrm{Si}$ and diamond are shown in Fig. 2 in comparison with experimental results. ${ }^{16,1}$ Our calculations give a very good description of the thermal expansion in both $\mathrm{Si}$ and diamond. In particular, the low-temperature negative thermal-expansion behavior in $\mathrm{Si}$ and the absence of negative thermal expansion in diamond are well reproduced.

From Eq. (1), the thermal-expansion coefficient can be related to the Grüneisen parameters of the various phonon $\operatorname{modes}^{17}$

$$
\alpha=\frac{1}{3 B_{0} V} \sum_{i} \gamma_{i} h v_{i} \frac{d}{d T}\left\{\left[\exp \left(h v_{i} / k_{B} T\right)-1\right]^{-1}\right\}
$$

in which $\gamma_{i}=-d\left(\ln v_{i}\right) / d(\ln V)$ is the Grüneisen parameter for the $i$ th normal mode and $B_{0}$ is the bulk modulus.
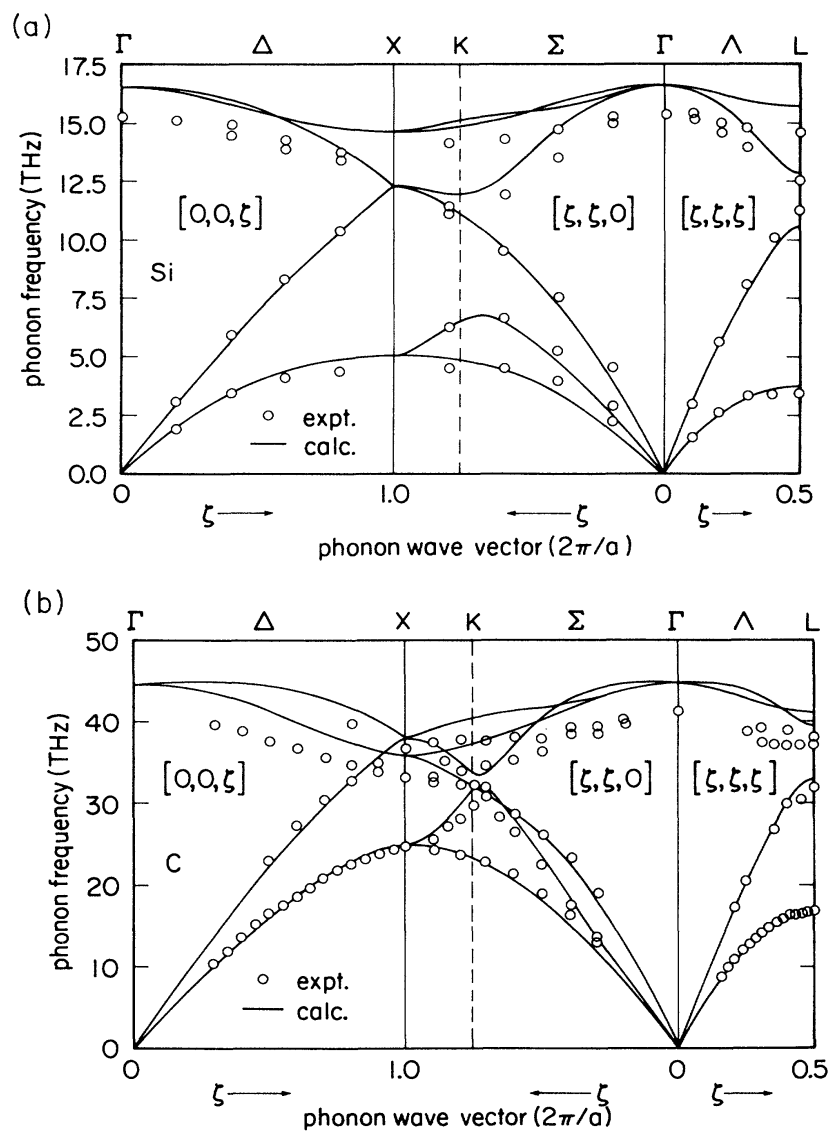

FIG. 1. Phonon dispersion curves for (a) Si and (b) diamond. Solid lines show the tight-binding calculation results. The experimental data (open circles) are from Ref. 1. (a)

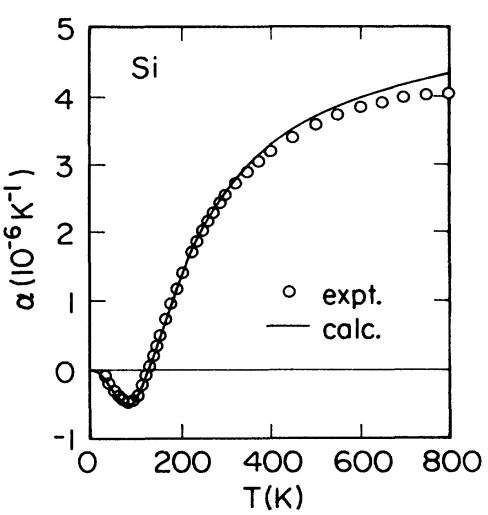

(b)

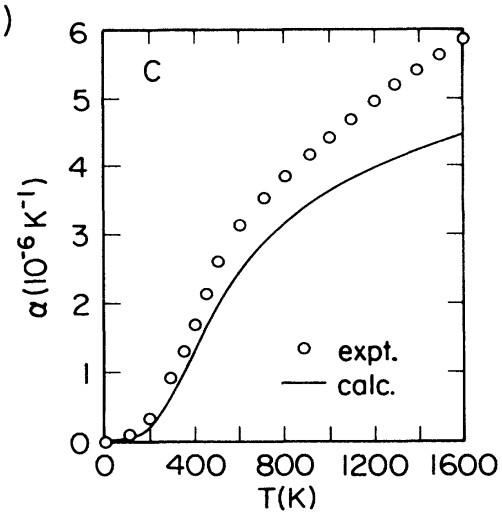

FIG. 2. Linear thermal-expansion coefficient of (a) Si and (b) diamond as a function of temperature. Results from our calculations are indicated by solid lines. Experimental data (open circles) are from Refs. 16 and 1, respectively.

Equation (3) shows that a negative thermal expansion can only occur when some of the phonon modes have negative Grüneisen parameters. Since the interatomic forces in the crystal usually weaken when the lattice expands, the Grüneisen parameters are usually positive for all the phonon modes in most materials. The results for the Grüneisen parameters of Si and diamond are plotted, respectively, in Figs. 3(a) and 3(b), where available experimental data ${ }^{1}$ are also displayed. The acoustic-phonon modes of $\mathrm{Si}$ behave anomalously: strong negative Grüeisen parameters are found for the TA branches especially near the Brillouin-zone boundaries $X$ and $L$. On the other hand, such behavior is not observed in the corresponding phonon branches of diamond.

The physical origin of the anomalous behavior of the TA modes in Si can be elucidated by a simple model where we consider only nearest-neighbor interactions. We show in Fig. 4 the patterns of atomic vibrations associated with the TA $(X)$ and the TA $(L)$ modes. The polarizations of these two modes are determined by the symmetry of the diamond lattice and are associated with pure bond-bending motion: the bonds between atoms are either undisturbed or are distorted by atomic motions perpendicular to the bonds. In such cases, we can describe the restoring force on the atoms as the sum of a centralforce part $\omega_{\text {cen }}$ and a noncentral angular part $\omega_{\text {noncen }}$ com- 
(a)
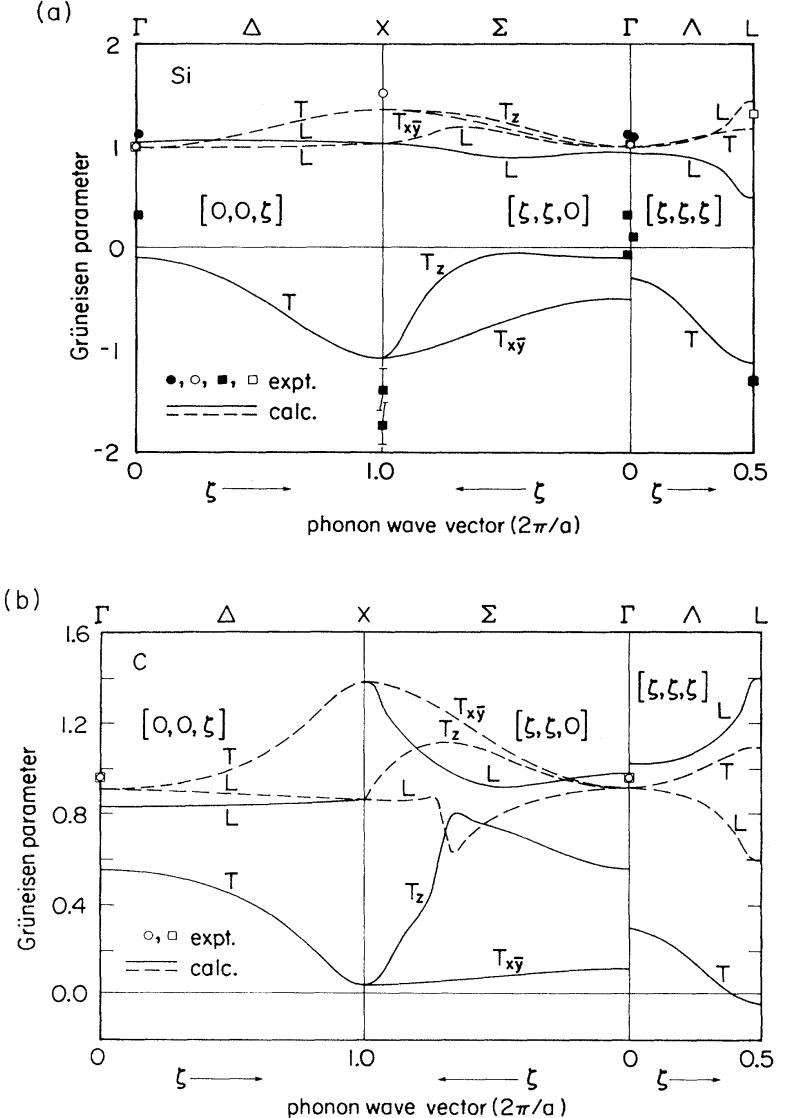

FIG. 3. Mode Grüneisen parameters for (a) Si and (b) diamond. Solid lines (acoustic modes) and dashed lines (optical modes) are results from our calculations. The experimental data $(\boldsymbol{\square}$ acoustic modes; $\bigcirc, \square$ optical modes; $\bigcirc, \bigcirc$ longitudinal; $\boldsymbol{\square}, \square$ transverse) are from Ref. 1.

ing from the directional covalent bonds stabilizing the diamond structure. Thus

$$
\omega_{0}^{2}=\omega_{\text {cen }}^{2}+\omega_{\text {noncen }}^{2} \text {. }
$$

The central-force contribution is well described by a spring model. For displacements perpendicular to a spring, the restoring force depends on the tension in the spring $\left(T_{s}\right)$. At the equilibrium volume $V_{0}$ the tension in

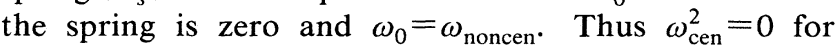
$V=V_{0}$. When $V>V_{0}$ the spring is stretched, $T_{s}>0$ giving a restoring force $\omega_{\text {cen }}^{2}>0$. When $V<V_{0}$, the spring is compressed, $T_{s}<0$ leading to $\omega_{\text {cen }}^{2}<0$. We can relate $\omega_{\text {cen }}^{2}$ to the bulk modulus as follows.

First, the central-force contribution to the force constant is related to the spring tension and the bond length d,

$$
m \omega_{\mathrm{cen}}^{2}=2 C T_{s} / d,
$$

where $C$ takes into account the number of bonds bent by the phonon distortion [ $C$ equals 1 for $\mathrm{TA}(L)$ and 2 for $\mathrm{TA}(X)]$ and $m$ is the mass of atom.

Now $T_{s}$ is related to the spring constant $k$ and the de- (a)

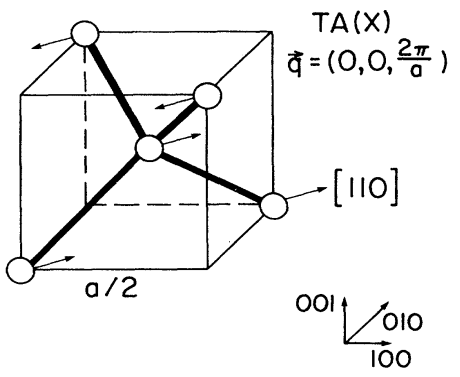

(b)

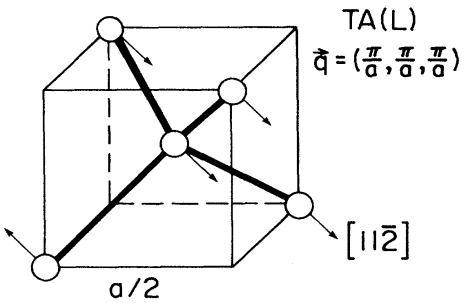

FIG. 4. Vibrational motion corresponding to the (a) TA $(X)$ mode and (b) TA $(L)$ mode in the diamond structure. The displacements of the ions are along the [110] direction in (a) and along the $[11 \overline{2}]$ direction in (b).

viation of the bond length from its equilibrium value $d_{0}$. Then $k$ and $d-d_{0}$ can be related to the bulk modulus $B_{0}$ and $\Delta V / V_{0}$. Thus,

$$
T_{s}=k\left(d-d_{0}\right),
$$

and

$$
2 \times \frac{1}{2} k\left(d-d_{0}\right)^{2}=\frac{1}{2} B_{0}\left(\frac{\Delta V}{V_{0}}\right)^{2} V_{0} .
$$

Finally,

$$
\gamma_{\text {cen }}=-\frac{d\left(\omega_{\text {cen }} / \omega_{0}\right)}{d(\ln V)}=-\frac{4 C}{\sqrt{3}} \frac{B_{0} d_{0}}{m \omega_{0}^{2}} .
$$

Thus $\gamma_{\text {cen }}$ is negative. This is the origin of the negative Grüneisen behavior of the TA modes in Si.

To get a complete description, we have to obtain the contribution from the angular forces. Using the bond orbital approximation, ${ }^{18}$ the change in covalent bond energy when the lattice is distorted with bond-bending motion is $d E \propto \eta \theta^{2}$ where $\eta$ is the tight-binding matrix element between bond orbitals on the two atoms at the ends of the bond and $\theta$ is the angle of rotation of the bond. Now $\eta$ varies as $1 / d^{2}$ and $\theta=2 u / d$ where $u$ is the atomic displacement, so $\omega_{\text {noncen }}^{2}$ varies as $1 / d^{4}$. Thus,

$$
\gamma_{\text {noncen }}=-d\left(\ln \omega_{\text {noncen }}\right) / d(\ln V)=\frac{2}{3} .
$$

Within our simple model, the Grüneisen parameters of the $\mathrm{TA}(X)$ and $\mathrm{TA}(L)$ modes can be written as

$$
\gamma=\frac{2}{3}-\frac{4 C}{\sqrt{3}} \frac{B_{0} d_{0}}{m \omega_{0}^{2}} .
$$

By using the bulk moduli and phonon frequencies from our tight-binding calculations, we can estimate from Eq. 
(10) the $\mathrm{TA}(X)$ and $\mathrm{TA}(L)$ mode Grüneisen parameters of $\mathrm{Si}$, diamond, and $\mathrm{Ge}$. The results are listed in Table I. Our model predicts that the TA modes have negative Grüneisen behavior in $\mathrm{Si}$ and $\mathrm{Ge}$ but not in diamond. The reason for the difference is that the directional covalent bonding is much stronger in diamond than in Si. Thus the angular forces are dominant in diamond and our analysis indicates that noncentral forces favor positive Grüneisen behavior. For negative thermal expansion, the angular forces should be weak but not absent, because otherwise the diamond structure would not be stable at all.

The above model can also be applied to the discussion of thermal expansion of zinc-blende semiconductors, although the analysis will be much more complicated because of the unequal masses of the atoms in the zincblende structure. However, in such cases, we expect tight-binding calculations will still give accurate answers.

In summary, we have shown that detailed calculations based on a simple tight-binding model can provide accurate thermal expansion results (for $\mathrm{Si}$ and diamond). We have given a microscopic analysis of the origin of the anomalous mode $[\mathrm{TA}(X)$ and TA $(L)]$ Grüneisen parameters in $\mathrm{Si}$. Our analysis provides a simple explanation of the negative thermal-expansion behavior in $\mathrm{Si}$ and the difference in thermal-expansion behavior of diamond and Si. We hope our work will provide a basis for consider-
TABLE I. The Grüneisen parameters $\gamma_{\mathrm{TA}(X)}$ and $\gamma_{\mathrm{TA}(L)}$ for $\mathrm{Si}$, diamond, and $\mathrm{Ge}$ are calculated from Eq. (10). The estimated $\gamma_{\mathrm{TA}(X)}$ and $\gamma_{\mathrm{TA}(L)}$ are compared with experimental data (Ref. 1) and tight-binding calculation results.

\begin{tabular}{llcc}
\hline & \multicolumn{1}{c}{$\mathrm{Si}$} & Diamond & $\mathrm{Ge}$ \\
\hline$\gamma_{\mathrm{TA}(X)}(T B$ calc. $)$ & -1.08 & 0.042 & \\
$\gamma_{\mathrm{TA}(X)}$ (expt.) & -1.4 & & -1.53 \\
$\gamma_{\mathrm{TA}(X)}$ [Eq. $\left.(10)\right]$ & -1.67 & 0.017 & -1.08 \\
& & & \\
$\gamma_{\mathrm{TA}(L)}(T B$ calc.) & -1.15 & -0.047 & \\
$\gamma_{\mathrm{TA}(L)}$ (expt.) & -1.3 & & -0.4 \\
$\gamma_{\mathrm{TA}(L)}$ [Eq. $\left.(10)\right]$ & -1.54 & -0.060 & -0.77 \\
\hline
\end{tabular}

ing thermal-expansion behavior in more complicated materials such as amorphous semiconductors, graphitic fibers, and polymer chains.

Ames Laboratory is operated for the U.S. Department of Energy by Iowa State University under Contract No. W-7405-ENG-82. This investigation was supported by the Director for Energy Research, Office of Basic Energy Sciences and by the Air Force Office of Scientific Research.
${ }^{1}$ Intrinsic Properties of Group IV Elements and III-V, II-VI, and I-VII Compounds, edited by D. Madelung, LandoltBörnstein, New Series, Group III, Vol. 22a, Pt. 12,33,262,268,270,312 (Springer-Verlag, Berlin, 1987); Physics of Group IV Elements and III-V Compounds, edited by O. Madelung, Landolt-Börnstein, New Series, Group III, Vol. 17a, Pt. 107 (Springer-Verlag, Berlin, 1982), and references therein.

${ }^{2}$ C. A. Swenson, J. Phys. Chem. Ref. Data 12 (2), 179 (1983).

${ }^{3}$ G. K. White and M. L. Minges, Thermophysical Properties of Some Key Solids (Pergamon, New York, 1985).

${ }^{4}$ M. Okaji, Int. J. Thermophys. 9 (6), 1101 (1988).

${ }^{5}$ See, for example, T. H. K. Barron, J. G. Collins, and G. K. White, Adv. Phys. 29, 609 (1980).

${ }^{6}$ S. Biernacki and M. Scheffler, Phys. Rev. Lett. 63, 290 (1989).

${ }^{7}$ H. Wendel and R. M. Martin, Phys. Rev. B 19, 5251 (1979).

${ }^{8}$ M. T. Yin and M. L. Cohen, Phys. Rev. Lett. 45, 1004 (1980); Phys. Rev. B 26, 3259 (1982).
${ }^{9}$ C. Z. Wang, C. T. Chan, and K. M. Ho, Phys. Rev. B 39, 8586 (1989).

${ }^{10}$ A. Bienenstock and G. Burley, J. Phys. Chem. Solids 24, 1271 (1963); A. Bienenstock, Philos. Mag. 9, 755 (1964).

${ }^{11}$ See, for example, L. L. Boyer, Phys. Rev. Lett. 42, 584 (1979).

${ }^{12}$ D. J. Chadi and R. M. Martin, Solid State Commun. 19, 643 (1976).

${ }^{13}$ D. J. Chadi, Phys. Rev. B 29, 785 (1984).

${ }^{14}$ J. R. Chelikowsky and S. G. Louie, Phys. Rev. B 29, 3470 (1984).

${ }^{15}$ Details for the evaluation of the dynamical matrix for tightbinding models are described in O. L. Alerhand and E. J. Mele, Phys. Rev. B 35, 5533 (1987).

${ }^{16}$ H. Ibach, Phys. Status Solidi 31, 625 (1969).

${ }^{17}$ N. W. Ashcroft and N. D. Mermin, Solid State Physics (Holt, Rinehart and Winston, New York, 1976), p. 492.

${ }^{18}$ W. A. Harrison, Electronic Structure and The Properties of Solids (Freeman, San Francisco, 1980). 